> Des progrès considérables ont été accomplis au cours des dix dernières années dans la compréhension des bases génétiques de l'obésité et des complications métaboliques qui lui sont associées. Il est maintenant bien établi que les différentes formes d'obésité constituent des états ayant tendance à se concentrer dans les familles et que les ressemblances familiales qu'on observe sont en partie déterminées par les gènes. Le nombre de gènes potentiellement impliqués dans le développement de l'obésité ne cesse de croître. Cet article fait le point sur le rôle de l'hérédité dans l'obésité et sur les gènes et locus qui ont, à ce jour, été associés à l'obésité au sein de l'étude des familles de Québec. <

\section{Bases génétiques de l'obésité familiale au Québec}

Louis Pérusse, Claude Bouchard

\author{
L. Pérusse: \\ Division de kinésiologie, PEPS, \\ Département de médecine \\ sociale et préventive, \\ Faculté de médecine, \\ Université Laval, Sainte-Foy, \\ Québec GIK 7P4, Canada. \\ C. Bouchard: Pennington \\ Biomedical Research Center, \\ Baton Rouge, Louisiane, \\ États-Unis.
}

\section{Influence des facteurs génétiques sur l'obésité}

Louis.Perusse@kin.msp.ulaval.ca
Notre compréhension des bases génétiques et moléculaires de l'obésité et des complications métaboliques qui lui sont associées s'est considérablement accrue au cours des dix dernières années. Les progrès réalisés jusqu'en 1998 et fondés sur l'étude des familles de Québec (Quebec Family Study, QFS), avaient fait l'objet d'un $(\rightarrow) \mathrm{m} / \mathrm{s}$ $1998, n^{\circ} 14$, p. 914 article de synthèse publié à l'occcasion du $8^{e}$ Congrès international d'obésité $(\rightarrow)$ [1]. Depuis lors, les progrès les plus importants ont été réalisés sur le plan moléculaire avec l'identification d'un grand nombre de gènes et locus associés à l'obésité. La carte génétique de l'obésité publiée annuellement depuis 1997 reflète bien les progrès accomplis, le nombre de gènes et marqueurs moléculaires répertoriés étant passé de 24 dans la première édition à plus de 300 dans la plus récente [2]. Cette synthèse a pour objectif de faire le point sur le rôle de l'hérédité dans l'obésité et sur les gènes et locus qui, à ce jour, ont été reliés à l'obésité en se basant principalement sur les résultats de l'étude des familles de Québec (Quebec Family Study, QFS).
Même s'il est bien connu que l'obésité se constate au sein des familles par des ressemblances entre les divers membres, jusqu'à tout récemment, le risque d'obésité chez un individu présentant des antécédents familiaux d'obésité n'était pas clairement établi. Ce risque familial a été calculé sur l'indice de masse corporelle (IMC = poids $[\mathrm{kg}] /$ taille $\left[\mathrm{m}^{2}\right]$ ) et un indicateur d'obésité abdominale (rapport des plis adipeux du tronc [somme des plis sous-scapulaire et supra-iliaque] sur ceux des extrémités [somme des plis du biceps, du triceps et du mollet]), à partir des données d'une enquête canadienne sur la condition physique et le mode de vie regroupant 15245 participants [3, 4]. Les résultats obtenus ont révélé que la prévalence de l'obésité était 4 fois plus élevée au sein des familles d'individus obèses que dans l'ensemble de la population. Dans le cas de l'obésité abdominale, le risque familial atteignait une valeur de 5. De plus, lorsque ce risque était calculé en ne considérant que les époux au lieu de tous les membres de la famille (parents et enfants), celui-ci demeurait 
inchangé dans le cas de l'obésité tandis qu'il devenait nul dans le cas de l'obésité abdominale. Puisque les époux ne partagent pas de gènes en commun par descendance, ces résultats suggèrent que l'obésité abdominale est sans doute plus influencée par des facteurs génétiques que ne l'est le surplus pondéral. En définitive, ces résultats font penser qu'une histoire familiale positive (où figurent des individus obèses) doit être considérée comme un facteur de risque d'obésité.

Les données de l'étude PFS ont permis d'estimer l'importance des facteurs génétiques et environnementaux dans le risque familial d'obésité. L'étude QFS est une étude familiale prospective portant sur les causes et les conséquences de l'obésité [5]. Elle est actuellement constituée de 951 sujets provenant de 223 familles québécoises chez lesquels on a mesuré une série de phénotypes reliés à l'obésité et à la distribution du tissu adipeux, à la consommation alimentaire et aux dépenses d'énergie, aux facteurs de risque et de comorbidités de l'obésité, ainsi qu'aux habitudes de vie. Les informations ont été recueillies à plus d'une reprise faisant de l'étude QFS la seule étude épidémiologique familiale sur l'obésité avec recueil de données longitudinales. Les données de l'étude PFS ont permis de démontrer des coefficients d'héritabilité de l'ordre de $5 \%$ pour l'IMC et la quantité de graisse sous-cutanée, de $25 \%$ pour le pourcentage de graisse mesurée par pesée hydrostatique, de $30 \%$ à $40 \%$ pour divers indicateurs de la distribution du tissu adipeux sous-cutané et d'environ $50 \%$ pour la quantité de graisse viscérale mesurée par tomodensitométrie [1]. D’autres études portant sur les déterminants de l'obésité ont rapporté une héritabilité plus modérée de l'ordre d'environ $20 \%$ pour l'apport en macronutriments [6] et le niveau d'activité physique [7]. Dans les cas des phénotypes associés aux co-morbidités de l'obésité, des coefficients d'héritabilité de l'ordre $50 \%$ à $60 \%$ ont été obtenus pour les concentrations plasmatiques de lipides et de lipoprotéines [8] et de $20 \%$ à $50 \%$ pour la pression artérielle [9]. Des études plus récentes ont également démontré une contribution des facteurs génétiques dans les changements d'adiposité en relation avec l'âge. C'est ainsi qu'une étude basée sur les données longitudinales de PFS a établi que les changements survenus sur une période de 12 ans pour l'IMC et la graisse sous-cutanée étaient influencés par l'hérédité, dans des proportions respectives de $37 \%$ et $16 \%$ [10]. Ces résultats ont été confirmés dans une étude récente réalisée auprès de 1315 sujets provenant de 521 familles canadiennes testés en 1981 et en 1988 [11]. La contribution de l'hérédité pour les modifications de I'IMC et de l'adiposité sous-cutanée atteignait respectivement
$14 \%$ et $12 \%$. Ces résultats suggèrent que tant le surplus pondéral et l'obésité que les gains de masse corporelle et d'adiposité observés avec l'âge sont influencés, à des degrés divers, par les gènes.

L'obésité est associée à plusieurs états métaboliques qui sont influencés par l'hérédité. Les auteurs d'articles sur le sujet [12-15] indiquent des degrés d'héritabilité variant de $25 \%$ à $70 \%$ pour les concentrations de lipides et de lipoprotéines plasmatiques et pour divers phénotypes reliés au métabolisme du glucose et de l'insuline.

\section{Gènes et marqueurs moléculaires de l'obésité}

Bien que l'on estime qu'il pourrait exister plus de 300 gènes ou locus intervenant dans l'obésité [2], très peu d'entre eux ont été directement impliqués jusqu'à présent dans l'étiologie de l'obésité. Cependant, il existe de rares cas d'obésité dont la cause est monogénique, c'est-à-dire attribuable à une mutation dans un seul gène. À ce jour, moins de 100 individus dans le monde ont été répertoriés [2] et, dans ces cas, les sujets sont généralement atteints d'une forme sévère et précoce d'obésité. Dans ces formes monogéniques d'obésité, un total de 36 mutations au sein de 6 gènes différents ont été décrites: gènes de la pro-hormone convertase-1 (PCSK1, 1 cas), de la leptine ( $L E P, 6$ cas), du récepteur de la leptine ( $L E P R, 3$ cas), de la pro-opiomélanocortine (POMC, 2 cas), du récepteur aux mélanocortines de type 4 (MC4R, 73 cas) et du gène SIMI (single-minded homolog 1, 1 cas), un gène codant pour un facteur de transcription.

L'identification des gènes associés à l'obésité chez l'humain repose sur deux stratégies principales: les études d'association et les études de liaison (linkage). Les études d'association consistent à comparer les fréquences alléliques et/ou génotypiques entre des groupes de sujets divergents pour un phénotype donné, ou à tester les différences phénotypiques entre individus de génotypes différents pour un polymorphisme d'un gène candidat. Un total de 71 gènes candidats ont été associés de façon positive, jusqu'à ce jour, dans au moins une étude, avec un phénotype d'obésité [2]. Parmi ces gènes, 15 ont ont été associés de façon positive à des phénotypes reliés à l'obésité ou à ses complications métaboliques au sein de l'étude PFS. Le Tableaul présente la liste de ces gènes et des phénotypes impliqués. Deux de ces gènes, le gène du récepteur des glucocorticoïdes $(G R L)$ et le gène du récepteur $\alpha 2$-adrénergique (ADRA2A), montrent des associations à la fois avec des phénotypes d'obésité et de complications métaboliques. D'autres, comme les gènes HSD3B1, $U C P I$ et $G R L$, montrent des associations avec les gains d'adiposité en relation avec l'âge. 
Les études de liaison sont généralement réalisées à l'aide de marqueurs génétiques anonymes plutôt que sur des gènes candidats. Lorsque ces études de liaison sont réalisées avec une série de marqueurs anonymes choisis de façon à couvrir l'ensemble du génome humain, elles constituent un criblage génomique (genome scan). Ces criblages génomiques permettent de repérer des locus associés à un caractère quantitatif ou trait quantitatif (quantitative trait locus, $\mathrm{PTL}$ ), c'est-à-dire des régions du génome contenant des gènes susceptibles d'influencer le trait étudié. Les régions ainsi localisées peuvent par la suite être étudiées de façon plus approfondie pour identifier les gènes responsables des liaisons génétiques avec le trait quantitatif. Un très grand nombre de criblages génomiques mettant en corrélation des phénotypes d'obé-

\begin{tabular}{|c|c|c|c|c|}
\hline \multirow[t]{2}{*}{ GÈNE } & \multirow[t]{2}{*}{ LOCALISATION } & \multicolumn{2}{|l|}{ PHÉNOTYPE } & \multirow[t]{2}{*}{ RÉFÉRENCES } \\
\hline & & Obésité & Complication métabolique & \\
\hline$L E P R$ & lp31 & Masse maigre & & [21] \\
\hline HSD3BI & lp13.1 & Gain de graisse sous-cutanée avec l'âge & & [22] \\
\hline ATPIA2 & $1 q 21-q 23$ & $\begin{array}{l}\text { Pourcentage de graisse; } \\
\text { quotient respiratoire }\end{array}$ & & {$[23,24]$} \\
\hline GHRL & $3 p 26-p 25$ & $\begin{array}{l}\text { Masse grasse; graisse viscérale; } \\
\text { quotient respiratoire }\end{array}$ & & [25] \\
\hline PPARG & $3 p 25$ & $\begin{array}{l}\text { IMC; masse grasse; } \\
\text { circonférence de la taille; } \\
\text { graisse sous-cutanée }\end{array}$ & & [26] \\
\hline$U C P 1$ & $4 q 28-q 31$ & Gain de masse grasse avec l'âge & & [27] \\
\hline GRL & $5 q 31-q 32$ & $\begin{array}{l}\text { Graisse viscérale; } \\
\text { gain de graisse sous-cutanée avec l'âge }\end{array}$ & HDL-C & {$[28,29]$} \\
\hline ADRB2 & $5 q 31-q 32$ & & CHOL, LDL-CHOL & {$[30]$} \\
\hline$L P L$ & $8 p 22$ & & VLDL-CHOL, TG, VLDL-TG, HDL-C & {$[29,31]$} \\
\hline ADRB3 & $8 p 12-p 11.2$ & Graisse sous-cutanée & & {$[30]$} \\
\hline ADRA2A & $10 q 24-q 26$ & $\begin{array}{l}\text { Ratio des plis du tronc sur plis des hanches; } \\
\text { graisse abdominale sous-cutanée }\end{array}$ & $\begin{array}{l}\text { Aire d'insuline suite à un test } \\
\text { de tolérance au glucose }\end{array}$ & {$[29,30,32]$} \\
\hline SURI & $11 p 15.1$ & & $\begin{array}{l}\text { Concentrations plasmatiques } \\
\text { de C-peptide à jeun et en réponse } \\
\text { à un test de tolérance au glucose }\end{array}$ & [33] \\
\hline UCP3 & $11 q 13$ & $\begin{array}{l}\text { IMC, pourcentage de graisse; } \\
\text { graisse sous-cutanée; leptine }\end{array}$ & & [34] \\
\hline$M C 4 R$ & $18 q 22$ & $\begin{array}{l}\text { Pourcentage de graisse; } \\
\text { masse grasse; masse maigre }\end{array}$ & & [35] \\
\hline MC3R & $20 q 13.2-q 13.3$ & $\begin{array}{l}\text { Pourcentage de graisse; } \\
\text { masse grasse; graisse abdominale }\end{array}$ & & [36] \\
\hline
\end{tabular}

Tableau I. Liste des gènes associés à l'obésité et à ses complications métaboliques au sein de l'étude des familles de Québec. LEPR: récepteur de la leptine; HSD3B1 : 3- $\beta$-hydroxy-stéroïde déshydrogénase; ATP1A2: sodium-potassium adénosine triphosphatase $\alpha-2$; GHRL: ghréline; PPAR $\gamma$ : peroxisome proliferative activated receptor $\gamma$; UCP1 : protéine découplante- 1 ; GRL: récepteur des glucocorticoïdes; $A D R B 2$ : récepteur adrénergique- $\beta 2$; LPL: lipoprotéine lipase; ADRB3: récepteur adrénergique- $\beta 3$; ADRA2A : récepteur adrénergique- $\alpha 2$; SUR1 : récepteur-1 de la sulfonylurée; UCP3: protéine découplante-3; MC4R: récepteur de la mélanocortine 4; MC3R: récepteur de la mélanocortine 3 . 
sité ont été réalisés. Selon la carte des gènes de l'obésité la plus récente [2], un total de 29 criblages génomiques de l'obésité entrepris au sein d'une quinzaine de populations différentes avec des phénotypes comme l'obésité, I'IMC, la composition corporelle (pourcentage de graisse et masse grasse), le rapport des circonférences de la taille et des hanches, la graisse abdominale, la dépense énergétique quotidienne, le quotient respiratoire, des hormones du tissu adipeux telles la leptine et l'adiponectine, ont révélé l'existence de 68 QTL d'obésité distribués sur tous les chromosomes, à l'exception des chromosomes 21 et y [2]. Quatre criblages génomiques ont été effectués jusqu'à présent au sein de l'étude QFS: un sur la masse maigre [16], un sur la quantité de graisse abdominale mesurée par tomodensitométrie et ajustée pour la masse grasse [17], un sur les pressions artérielles systolique et diastolique [18] et un sur la taille des particules de LDL-cholestérol [19]. Le Tableau // présente la liste des QTL identifiés à partir de ces études et l'analyse de liaison par lod score pour chacun de ces QTL. Ces études ont permis de mettre en évidence 3 QTL pour la masse maigre (7p, 15q et $18 q$ ), 9 QTL pour l'obésité abdominale ( $1 p, 4 p, 4 q$, $7 q, 9 q, 12 q 22,12 q 24,13 q$ et $17 q), 7$ QTL pour la tension artérielle (1p, 2p, 2q, 5p, 7q, 8q, 19p) et 6 QTL pour la taille des particules de LDL-cholestérol (lp, 2q, 4p, 5q, $14 q, 17 q)$. Il est intéressant de noter que deux des (TTL associés à l'obésité abdominale (4p15 et 17q21) sont également associés à la tailles des particules de LDLcholestérol. Puisque les particules de LDL de petite taille sont liées à un risque accru de maladie coronarienne, ces résultats suggèrent que ces deux régions $\mathrm{du}$ génome contiennent des gènes qui ne sont pas étrangers au risque que survienne une maladie coronarienne chez les patients atteints d'obésité abdominale.

\section{Discussion}

Cette synthèse démontre l'ampleur des progrès qui ont été réalisés au cours des dernières années dans l'identification des gènes associés à l'obésité. En dépit des progrès considérables qui ont été accomplis, il est important de prendre conscience que les stratégies utilisées pour repérer les gènes associés à des traits complexes comme l'obésité et ses complications métaboliques (pour revue, voir [20]), ont des limitations et peuvent donner lieu à de nombreux faux positifs. Il est clair que parmi tous les gènes circonscrits à ce jour, certains vont s'avérer plus importants que d'autres dans l'étiologie de l'obésité. Une façon d'établir l'importance des gènes en cause consiste à identifier ceux qui ont fait l'objet de réplications au sein de diffé- rentes populations, c'est-à-dire celles pour lesquels des résultats positifs ont été rapportés dans plusieurs études. Ainsi, parmi les 71 gènes montrant des associations positives avec des phénotypes d’obésité [2],

\begin{tabular}{|c|c|c|}
\hline Localisation & Marqueur & Lod score \\
\hline \multicolumn{3}{|c|}{ Masse maigre } \\
\hline $7 p 15.3$ & D7S1808 & 2,7 \\
\hline $15 q 25-q 26$ & IGFIR & 3,6 \\
\hline $18 q 12$ & D18S535 & 3,6 \\
\hline \multicolumn{3}{|c|}{ Obésité abdominale } \\
\hline lpll.2 & DIS534 & 2,3 \\
\hline $4 q 32.1$ & D4S2417 & 1,8 \\
\hline $4 p 15.1$ & D4S2397 & 2,3 \\
\hline $7 q 31.1$ & D7S1875 & 2,0 \\
\hline $9 q 22.1$ & D9S1122, D9S257 & $2,1-2,3$ \\
\hline $12 q 22-q 23$ & IGFl & 1,9 \\
\hline \multirow[t]{3}{*}{$12 q 24.3$} & D12S2078 & \\
\hline & D12S1045 & 1,5 \\
\hline & & 2,9 \\
\hline $13 q 34$ & D13S285 & 1,9 \\
\hline \multirow[t]{3}{*}{$17 q 21.1-q 21.3$} & D17S2180 & \\
\hline & D17S1301 & 2,2 \\
\hline & & 2,2 \\
\hline \multicolumn{3}{|c|}{ Pression artérielle } \\
\hline lp22.1 & D1S551 (PAS) & 1,8 \\
\hline $2 p 11.1$ & D2S1790 (PAS, PAD) & $1,3-2,2$ \\
\hline $2 q 11.2$ & D2S2972 (PAS) & 2,0 \\
\hline $5 p 13.3$ & D5S1986 (PAS) & 2,5 \\
\hline $7 q 32.1$ & D7S5307 (PAS) & 1,5 \\
\hline $8 q 21.1$ & CRH (PAS) & 2,1 \\
\hline $19 p 13.3$ & D19S247 (PAS) & 2,1 \\
\hline \multicolumn{3}{|c|}{ Taille des particules de LDL-cholestérol } \\
\hline lp31 & LEPR & 2,6 \\
\hline $2 q 33.2$ & D2S1384 & 2,3 \\
\hline $4 p 15.2$ & D4S2397 & 2,1 \\
\hline $5 q 12.3$ & D5S1501 & 2,1 \\
\hline $14 q 13.1$ & D14S53 & 1,7 \\
\hline $17 q 21.33$ & D17S1301 & 6,8 \\
\hline
\end{tabular}

Tableau II. Résumé des études de criblage génomique réalisées au sein de l'étude des familles de Québec pour des phénotypes associés à l'obésité et à ses complications métaboliques. Quatre criblages génomiques ont été effectués au sein de cette étude : un sur la masse maigre [16], un sur la quantité de graisse abdominale mesurée par tomodensitométrie et ajustée pour la masse grasse [17], un sur les pressions artérielles systolique et diastolique [18] et un sur la taille des particules de LDL-cholestérol [19]. La liste des locus associés à un trait quantitatif (quantitative trait locus ou PTL) identifiés à partir de ces études et le lod score pour chacun de ces PTL sont présentés. PAS: pression artérielle systolique; PAD: pression artérielle diastolique. 
moins du tiers, soit 21, ont fait l'objet de réplications dans au moins trois études différentes. Parmi ces 21 gènes, 9 ont été associés à l'obésité dans l'étude QFS (gènes $L E P R, P P A R \gamma, U C P 1, G R L, A D R B 2, L P L, A D R B 3$, $A D R A 2 A$ et UCP3 du Tableau I) et 12 autres (gènes $A G T$, $A P O B, T N F, L E P, I G F 2, I N S, U C P 2, D R D 2, G N B 3, L D L R$, LIPE, HTR2C*) ont montré des associations positives au sein d'autres populations. Les réplications sont encore moins nombreuses pour les QTL identifiés à partir des différents criblages génomiques puisque seulement 6 des 68 QTL d'obésité répertoriés à ce jour [2], soit moins de $10 \%$, ont été identifiés dans au moins 3 études. Les QTL ayant fait l'objet de ces réplications sont situés sur les chromosomes 3q27, 6p21.3-p21.1, $10 p 12-p 11,11 q 23-q 24,17 p 12$ et $18 q 21$. Ces régions du génome humain sont donc porteuses de gènes susceptibles de jouer un rôle important dans le développement de l'obésité et de ses complications métaboliques au sein d'une fraction importante de la population. Des études approfondies de ces régions du génome devront être effectuées afin de repérer les gènes en cause.

\section{Conclusions}

L'alimentation riche en lipides et le mode de vie sédentaire qui caractérisent les sociétés modernes jouent un rôle important dans l'augmentation de la prévalence des cas d'obésité observés partout à travers le monde. Cependant, il ne fait maintenant plus de doute que nous ne sommes pas tous égaux face à ces facteurs de risque et que nos gènes sont responsables de la façon dont nous nous adaptons à l'environnement « obésogène » dans lequel nous vivons. Même si nos connaissances sur les bases génétiques de l'obésité se sont beaucoup améliorées au cours des dix dernières années, les gènes responsables de cette susceptibilité génétique restent
*AGT: angiotensinogène; $A P O B$ : apolipoprotéine B; TNF: tumor necrosis factor; LEP: leptine; IGF2: insulin-like growth factor; INS: insuline; UCP2: uncoupling protein 2; DRD2: dopamine receptor D2; GNB3: guanine nucleotide binding protein (G protein) $\beta$-polypeptide 3; LDLR : low density lipoprotein receptor; LIPE: lipase, hormone sensitive; HTR2C: 5 -hydroxytryptamine (serotonin) receptor $2 \mathrm{C}$.

encore à définir. L'identification de ces gènes permettra de mieux cerner l'étiologie de l'obésité et de ses complications métaboliques, de repérer les individus ou les groupes à risque, en fonction de leur profil génétique et de développer des stratégies de prévention et de traitement individualisées. $\diamond$

\section{SUMMARY}

Genetics of obesity and metabolic complications in the Quebec Family Study

Considerable progress has been accomplished over the past 10 years in the understanding of the genetic basis of obesity and its related metabolic complications. It is now well established that obesity aggregates within families and that genes are partly responsible for this familial aggregation. The number of genes potentially involved in obesity continues to grow. This review summarizes the evidence accumulated so far regarding the contribution of genetic factors in obesity and the number of gene and loci linked to obesity in the Quebec Family Study. $\bullet$

\section{RÉFÉRENCES}

1. Pérusse L, Chagnon YC, Rice T, Rao DC, Bouchard C. L'épidémiologie génétique et la génétique moléculaire de l'obésité: les enseignements de l'étude des familles de Québec. Med Sci 1998; 14: 914-24.

2. Chagnon $Y C$, Rankinen $T$, Snyder દદ, Weisnagel SJ, Pérusse L, Bouchard C. The human obesity gene map: the 2002 update. Obes Res 2003; 11: 313-67.

3. Katzmarzyk PT, Pérusse L, Rao DC, Bouchard C. Familial risk of obesity and central adipose tissue distribution in the general Canadian population. Am J Epidemiol 1999; 149: 933-42.

4. Katzmarzyk PT, Pérusse L, Rao DC, Bouchard C. Familial risk of overweight and obesity in the Canadian population using the WHO/NIH criteria. Obes Res $2000 ; 8$ : 194-7.
5. Bouchard C. Genetic epidemiology, association and sib-pair linkage: results from the Quebec Family Study. In: Bray GA, Ryan DH, eds. Molecular and genetic aspects of obesity. Baton Rouge: Louisiana State University Press, 1996: 470-81.

6. Pérusse L, Tremblay A, Leblanc $C$, et al. Familial resemblance in energy intake: contribution of genetic and environnemental factors. Am J Clin Nutr 1988; 47: 629-35.

7. Simonen RL, Perusse L, Rankinen T, Rice T, Rao DC, Bouchard C. Familial aggregation of physical activity levels in the Quebec Family Study. Med Sci Sports Exerc 2002; 34: 1137-42.

8. Pérusse L, Després JP, Tremblay A, et al. Genetic and environmental determinants of serum lipids and lipoproteins in French Canadian families. Arteriosclerosis 1989; 9: 308-18. 
9. Rice T, Vogler GP, Perusse L, Bouchard C, Rao DC. Cardiovascular risk factors in a French Canadian population: resolution of genetic and familial environmental effects on blood pressure using twins, adoptees, and extensive information on environmental correlates. Genet Epidemiol 1989; 6: 571-88.

10. Rice T, Perusse L, Bouchard C, Rao DC. Familial aggregation of body mass index and subcutaneous fat measures in the longitudinal Quebec family study. Genet Epidemiol 1999; 16: 316-34.

11. Hunt MS, Katzmarzyk PT, Perusse L, Rice T, Rao DC, Bouchard C. Familial resemblance of 7 -year changes in body mass and adiposity. Obes Res 2002; 10: 507-17.

12. Bouchard C. Genetics and the metabolic syndrome. Int J Obes Relat Metab Disord 1995; 19 (suppl 1): S52-9.

13. Groop L. Genetics of the metabolic syndrome. BrJ Nutr 2000; 83 (suppl1): S39-48.

14. Ukkola 0 , Bouchard C. Clustering of metabolic abnormalities in obese individuals: the role of genetic factors. Ann Med 2001; 33: 79-90.

15. Bougneres P. Genetics of obesity and type 2 diabetes: tracking pathogenic traits during the predisease period. Diabetes 2002; 51 (suppl3): S295-303.

16. Chagnon YC, Borecki IB, Perusse L, et al. Genomewide search for genes related to the fat-free body mass in the Quebec family study. Metabolism 2000; 49: 203-7.

17. Pérusse $L$, Rice $T$, Chagnon $y$, et al. A genome-wide scan for abdominal fat assessed by computed tomography in the Quebec Family Study. Diabetes 2001; 50: 614-21.
18. Rice T, Rankinen T, Province MA, et al. Genome-wide linkage analysis of systolic and diastolic blood pressure: the Quebec Family Study. Circulation 2000; 102: 1956-63.

19. Bossé $Y$, Pérusse L, Després $J P$, et al. Evidence for a major quantitative trait locus on chromosome $17 q 21$ affecting lowdensity lipoprotein peak particle diameter. Circulation 2003; 107: 2361-8.

20. Comuzzie AG, Williams JT, Martin LJ, Blangero J. Searching for genes underlying normal variation in human adiposity. J Mol Med 2001; 79: 57-70.

21. Chagnon $Y C$, Chung WK, Pérusse L, Chagnon M, Leibel RL, Bouchard C. Linkages and associations between the leptin receptor (LEPR) gene and human body composition in the Quebec Family Study. Int J Obes Relat Metab Disord 1999; 23: 278-86.

22. Vohl MC, Dionne FT, Pérusse L, Dériaz 0 , Chagnon $M$, Bouchard C. Relation between BgIII polymorphism in $3 \beta$-hydroxysteroid dehydrogenase gene and adipose-tissue distribution in human. Obes Res 1994; 2: 444-9.

23. Dériaz 0, Dionne F, Pérusse $L$, et al. DNA variation in the genes of the $\mathrm{Na}$, K-adenosine triphosphatase and its relation with resting metabolic rate, respiratory quotient, and body fat. J Clin Invest 1994; 93: 838-43.

24. Katzmarzyk PT, Rankinen T, Pérusse L, et al. Linkage and association of the sodium potassiumadenosine triphosphatase $\alpha 2$ and $\beta$ genes with respiratory quotient and resting metabolic rate in the Quebec Family Study. J Clin Endocrinol Metab 1999; 84: 2093-7.
25. Ukkola 0, Ravussin $\varepsilon$, Jacobson P, et al. Role of ghrelin polymorphisms in obesity based on three different studies. Obes Res 2002; 10: 782-91.

26. Robitaille J, Despres JP, Perusse L, Vohl MC. The PPAR- $\gamma$ P12A polymorphism modulates the relationship between dietary fat intake and components of the metabolic syndrome: results from the Quebec Family Study. Clin Genet 2003; 63: 109-16.

27. Oppert JM, Vohl MC, Chagnon M, et al. DNA polymorphism in the uncoupling protein (UCP) gene and human body fat. Int J Obes Relat Metab Disord 1994; 18: 526-31.

28. Buemann B, Vohl MC, Chagnon $M$, et al. Abdominal visceral fat is associated with a Bcll restriction fragment length polymorphism at the glucocorticoid receptor gene locus. Obes Res 1997; 5: 186-92.

29. Ukkola 0, Pérusse L, Weisnagel SJ, et al. Interactions among the glucocorticoid receptor, lipoprotein lipase, and adrenergic receptor genes and plasma insulin and lipid levels in the Quebec Family Study. Metabolism 2001; 50: 246-52.

30. Ukkola 0, Rankinen T, Weisnagel SJ, et al. Interactions among the $\alpha 2-, \beta 2-$, and $\beta 3$-adrenergic receptor genes and obesity-related phenotypes in the Quebec Family Study. Metabolism 2000; 49: 1063-70.

31. Ukkola 0, Garenc C, Pérusse $L$, et al. Genetic variation at the lipoprotein lipase locus and plasma lipoprotein and insulin levels in the Quebec Family Study. Atherosclerosis 2001; 158: 199-206.
32. Oppert JM, Tourville J, Chagnon $M$, et al. DNA polymorphisms in $\alpha 2$ - and $\beta 2$-adrenoceptor genes and regional fat distribution in humans: association and linkage studies. Obes Res 1995; 3: 249-55.

33. Weisnagel SJ, Rankinen T, Nadeau A, et al. Decreased fasting and oral glucose stimulated C-peptide in nondiabetic subjects with sequence variants in the sulfonylurea receptor 1 gene. Diabetes 2001; 50: 697-702.

34. Lanouette CM, Giacobino JP, Perusse L, et al. Association between uncoupling protein 3 gene and obesity-related phenotypes in the Quebec Family Study. Mol Med $2001 ; 7: 433-41$.

35. Chagnon YC, Chen WJ, Pérusse $L$, et al. Linkage and association studies between the melanocortin receptors 4 and 5 genes and obesity-related phenotypes in the Québec Family Study. Mol Med 1997; 3: 663-73.

36. Boucher N, Lanouette CM, Larose M, Pérusse L, Bouchard C, Chagnon YC. A +2138InsCAGACC polymorphism of the melanocortin receptor 3 gene is associated in human with fat level and partitioning in interaction with body corpulence. Mol Med 2002; 8: 158-65.

\section{TIRÉS À PART}

L. Pérusse 